www.jmscr.igmpublication.org

Impact Factor 5.84

Index Copernicus Value: 83.27

ISSN (e)-2347-176x ISSN (p) 2455-0450

crossref DOI:_https://dx.doi.org/10.18535/jmscr/v5i3.85

Journal Of Medical Science And Clinical Research

\title{
Validity of Sonourethrography in Assessing Anterior Urethral Strictures in Males
}

Authors

\author{
Dr Josey Verghese, Dr Ajay Alex, Dr K.B. Salim
}

Department of Radiodignosis, Government T.D. Medical College, Alappuzha, Kerala, India

Corresponding Author

Dr Josey Verghese

Email: drjoseyverghese@yahoo.co.in

\begin{abstract}
Introduction: Stricture urethra in males is a common problem encountered by the radiologists. Retrograde urethrography and voiding cystourethrography have been the standard imaging studies for the evaluation of male anterior urethra, which involves the use of radiation and contrast medium to visualize luminal anatomy and give limited information about periurethral structures. The aim of the study was to assess sonourethrogram versus retrograde urethrogram, in the evaluation of male urethral strictures.

Materials and Methods: In this descriptive study 54 patients with obstructive lower urinary tract symptoms and suspected to be having stricture urethra were subjected to ascending urethrogram and sonourethrogram. The findings were recorded for comparison.

Results: Intraclass Correlation Coefficient and measure of agreement of kappa between retrograde urethrography and sono urethrography in the assessment of stricture length were 0.981 and 0.447 respectively. The sensitivity [100\% (95\% CI 84.6-100)], specificity [100\% (95\% CI 89.1-100)], positive predictive value [100\% (95\% CI 84.6-100)], and negative predictive value [100\% (95\% CI 89.1-100)], of sonourethrography in the detection of urethral strictures for its presence and correct site were also estimated. In addition, spongiofibrosis, and severity of stricture were diagnosed only by sonourethrogram.

Conclusion: Sonourethrogram is an effective alternative to retrograde urethrogram in the evaluation of stricture urethra. It is superior to retrograde urethrogram in the identification of spongiofibrosis and effective in getting a cross sectional image.

Keywords: anterior urethra, retrograde urethrography, sonourethrography, stricture.
\end{abstract}

\section{INTRODUCTION}

Symptoms attributable to urethral diseases cause significant physical disability and psychological trauma to the patients. So it is of utmost importance that they are diagnosed and treated in an effective manner. Stricture urethra in males is a common problem encountered by the urologists and radiologists. Because the male urethra is longer than that of the female and traverses through several anatomical structures such as the prostate, urogenital diaphragm, and corpus spongiosum of the penis, it is more vulnerable to injury and stricture than the female urethra. Urethral strictures can result from inflammatory, ischemic, or traumatic processes. These processes lead to scar tissue formation. Scar tissue contracts and reduces the calibre of the urethral lumen, causing resistance to the antegrade flow of urine. 
The term urethral stricture generally refers to the anterior urethra and is secondary to scarring in the spongy erectile tissue of the corpus spongiosum. A posterior urethral stricture is due to a fibrotic process that narrows the bladder neck and usually results from a distraction injury secondary to trauma or surgery, such as radical prostatectomy. For long urinary tract infections and sexually transmitted diseases were the leading causes of urethral strictures. With the advent of early and effective treatment ${ }^{1}$ for these diseases, they no longer are the predominant causes of urethral strictures. Blunt injury is by far the leading cause of urethral strictures in the modern era ${ }^{2,3}$. While posterior urethral strictures are almost always secondary to pelvic fractures, anterior urethral strictures are secondary to straddle injuries ${ }^{4}$. Patients with urethral strictures commonly present with obstructive voiding symptoms. These patients are further investigated by the radiologist to establish the presence of a urethral disease and if present to further characterize the lesion so as to provide valuable preoperative information for the surgeon. Dynamic retrograde urethrography supplemented by various antegrade techniques wherever necessary has been the gold standard for investigations of the male urethra. Iodinated contrast material is used for these studies .The term dynamic infers the motion of the contrast through the urethra at the time of exposure of the film. This is especially important while imaging the posterior urethra, which is in a collapsed state normally. However conventional radiographic technique has some drawbacks. The film gives information only in a two dimensional plane, provides no information regarding the periurethral fibrosis, exposes the patient to radiation and the stricture length assessment is inaccurate ${ }^{5}$. Highresolution ultrasound has revolutionized the imaging of the anterior urethra. High frequency linear probes are used for this purpose. Sonourethrogram has the potential to become an effective alternative technique for the evaluation of the anterior urethra. Ultrasound provides highresolution three-dimensional images and most of the drawbacks of conventional radiography are overcome. There is no radiation exposure. Periurethral fibrosis is accurately assessed and the stricture length is accurately determined ${ }^{6}$. So sonourethrogram has added a whole new dimension to the characterization of the stricture. Sonourethrographic characterization of stricture apart from the accurate localization of the stricture also gives accurate information of the length of the stricture, degree of occlusion and periurethral spongiofibrosis ${ }^{7}$. These additional informations are absolutely essential for the reconstructive urologist to plan the right surgical technique ${ }^{8}$. For a patient, to suffer from a urethral stricture is a traumatic experience but to suffer from a recurrent stricture is extremely unfortunate. In most cases, the recurrence of strictures is due to selection of a faulty surgical option ${ }^{9,10,11}$.This is due to limited preoperative information provided by a conventional retrograde urethrography. Preoperative sonourethrographic assessment provides vital information, which helps the surgeon in selecting the right surgical option, thus reducing the incidence of recurrence by a great deal. So the rationale of the present study is to reassess the usefulness of sonourethrography against conventional RGU in assessing the presence of stricture, stricture site, stricture length and also for identifying spongiofibrosis.

\section{MATERIALS AND METHODS}

This was a descriptive study with diagnostic test evaluation, done from January 2014- September 2015 in a tertiary care hospital. Total 54 patients were included, all male patients with symptoms suggestive of urethral obstruction coming to the department. Inclusion criteria included age more than 10 yrs. Patients who were unwilling to be part of the study and seriously ill patients, those suffering from urinary tract infections and psychiatric illnesses who could not be interviewed and not in a state of providing information, were excluded from the study. Interviewer administered questionnaire in which details like site, size, length of stricture and periurethral spongiofibrosis 
were collected. Ethical committee sanction was obtained. The subjects were explained about complications and benefits of the procedure and written informed consent were taken from all the study subjects before performing the study. All study subjects were subjected to first retrograde urethrogram and then sonourethrography. The subjects were placed in a supine position. Under aseptic techniques, 12-16 sized Foley's catheter was inserted into the urethra after applying 2\% Lignocaine jelly and the bulb inflated at the fossa navicularis with $1-2 \mathrm{ml}$ of saline. Then $10-15 \mathrm{ml}$ of urograffin(non ionic contrast agent) was injected into the catheter. The films were taken in both right and left posterior oblique positions after abduction and flexion of the leg in contact with the table. After the retrograde urethrography, sonourethrogram was performed with the patient placed in a supine position. About $15-20 \mathrm{ml}$ of normal saline was introduced through the Foley's catheter continuously with a syringe. The patient was asked to contract his pelvic muscles in an attempt to retain the saline in the urethra. Bed side ultrasound scan using a 5-13 $\mathrm{MHz}$ linear transducer was done. Multiple real time B mode images of the anterior urethra were obtained in both the longitudinal and transverse planes. Based on retrograde urethrography the site and length of the strictures were recorded by an independent observer so as to make sure the study was blinded and unbiased. Based on the sonourethrography, findings were sub classified based on site (penile, penobulbar, bulbar and bulbomembraneous urethra), length (The length of the rigid non distensible portion of the urethra was measured in millimetres), degree of stricture (Proportional comparison between the stricture segment and the normal luminal diameter were measured in crosssectional plane as (a) $<1 / 3$ lumen occluded, (b) $1 / 3$ - 1/2 lumen occluded and (c) >1/2 lumen occluded) and periurethral spongiofibrosis( Graded as (a) Mild-Mild periurethral reflectivity, (b) Moderate-Moderate periurethral reflectivity and (c) Severe-Severe periurethral reflectivity and posterior acoustic shadowing $)^{12,13,14}$. Data collected were entered into Microsoft Excel worksheet for statistical analysis. The frequency tables of different vaiables, Intraclass correlation coefficient and kappa statistics of agreement between retrograde urethrographic and sonourethrographic stricture lengths were assessed. Sensitivity, specificity, positive predictive value and negative predictive value of sonourethrography, taking retrograde urethrography as reference standard were also assessed.

\section{RESULTS}

Minimum age of study sample was 14 years and maximum age of study was 78 years. Mean age of the study sample was 55 years. Most number of the study sample belonged to the 51-70 yrs age group, accounting for $58 \%$ of the study sample. Least number of the study sample belonged to the 10-30 yrs age group, accounting for

$9 \%$ of the study sample.

Trauma (83\%) was by far the leading cause of anterior urethral strictures based on this study. The rest was attributed to UTI. Fourteen of the patients with anterior urethral strictures had no such previous surgical / interventional procedures done whereas eight had h/o surgical / interventional procedures.

Degree of occlusion and spongiofibrosis were two parameters that could be assessed with sonourethrography and not by conventional RGU. The degree of occlusion and spongiofibrosis as assessed by sonourethrography is summarised in Table No 1 and 2.

Site of stricture as assessed by the two modalities were compared which showed similar localisation in conventional RGU and sonourethrography (Table No : 3 )

The stricture length assessments by both the modalities were also assessed. In two of the cases the stricture length could not be assessed in its entire length as in both the cases, the stricture extended into the posterior urethra. This exposes one of the limitations of sonourethrography. There were significant differences in stricture length assessed by both the modalities. The stricture 


\section{JMSCR Vol||05||Issue||03||Page 18807-18813||March}

length was found to be more in the Sonourethrography as compared to the Retrograde urethrography. $14 \%$ of the cases with stricture showed no difference in stricture length between the two modalities with the remaining $86 \%$ showing stricture length more in the sonourethrogram compared to the retrograde urethrogram (Fig 1 ).

Sonourethrogram detected the stricture in all the 22 cases which were detected by retrograde urethrography as well (Table No: 4).Test of performance of sonourethrogram was done with a sensitivity, specificity, positive predictive value and negative predictive value of $100 \%$ (Table No: 5).

$\begin{array}{lrrr}\text { Intraclass } & \begin{array}{c}\text { Correlation } \\ \text { between }\end{array} & \text { Coefficient of } & 0.981 \\ \text { Retrograde } & \text { urethrogram } & \text { and }\end{array}$ Sonourethrogram in the assessment of stricture length was obtained. However, Cohen's kappa level of agreement between Retrograde urethrogram and Sono urethrogram in the assessment of stricture length was only 0.447 which was only moderate in agreement.

Table No 1: Degree of occlusion of strictures in sonourethrography

\begin{tabular}{|l|c|c|}
\hline Degree of occlusion & Total numbers & Percent \\
\hline Mild (Less than1/3 lumen occluded) & 7 & 32 \\
\hline Moderate (Between $1 / 3-1 / 2$ lumen occluded) & 7 & 32 \\
\hline Severe(more than $1 / 2$ lumen occluded) & 8 & 36 \\
\hline Total & 22 & 100 \\
\hline
\end{tabular}

Table No 2 : Strictures based on the degree of spongiofibrosis in sonourethrography

\begin{tabular}{|l|c|c|}
\hline Degree of spongiofibrosis & Frequency & Percent \\
\hline Normal-Mild & 10 & 45 \\
\hline Mild- Moderate & 5 & 23 \\
\hline Moderate-Severe & 7 & 32 \\
\hline Total & 22 & 100 \\
\hline
\end{tabular}

Table No 3: Comparison of the location of the stricture by both the modalities.

\begin{tabular}{|l|c|c|}
\hline Site & Retrograde urethrography & Sonourethrography \\
\hline Penile & 4 & 4 \\
\hline Bulbar & 9 & 9 \\
\hline Penobulbar & 2 & 2 \\
\hline Bulbo Membraneous & 7 & 7 \\
\hline Total & 22 & 22 \\
\hline
\end{tabular}

Table No 4: Comparison of positive and negative stricture cases by retrograde urethrography and sonourethrography.

\begin{tabular}{|c|c|c|}
\hline & RGU $^{\ddagger}$ Positive & RGU $^{\ddagger}$ Negative \\
\hline Sonourethrogram positive ${ }^{*}$ & 22 & 0 \\
\hline Sonourethrogram negative ${ }^{\dagger}$ & 0 & 32 \\
\hline \multicolumn{3}{|c|}{$\begin{array}{l}\text { Sonourethrogram positive cases means those cases which were positive for both stricture presence and stricture site } \\
\text { as same as in RGU. } \\
{ }^{*} \text { Sonourethrogram negative means those cases which were not conforming to the above criteria. } \\
{ }^{*} \text { RGU: Retrograde urethrogram. }\end{array}$} \\
\hline
\end{tabular}

Table No 5: Test of performance of sonourethrography, when RGU is taken as reference, in identifying strictures.

\begin{tabular}{|l|l|}
\hline Sensitivity & $100 \%(95 \%$ CI $84.6-100)$ \\
\hline Specificity & $100 \%(95 \%$ CI $89.1-100)$ \\
\hline Positive predictive value & $100 \%(95 \%$ CI $84.6-100)$ \\
\hline Negative predictive value & $100 \%(95 \%$ CI $89.1-100)$ \\
\hline
\end{tabular}




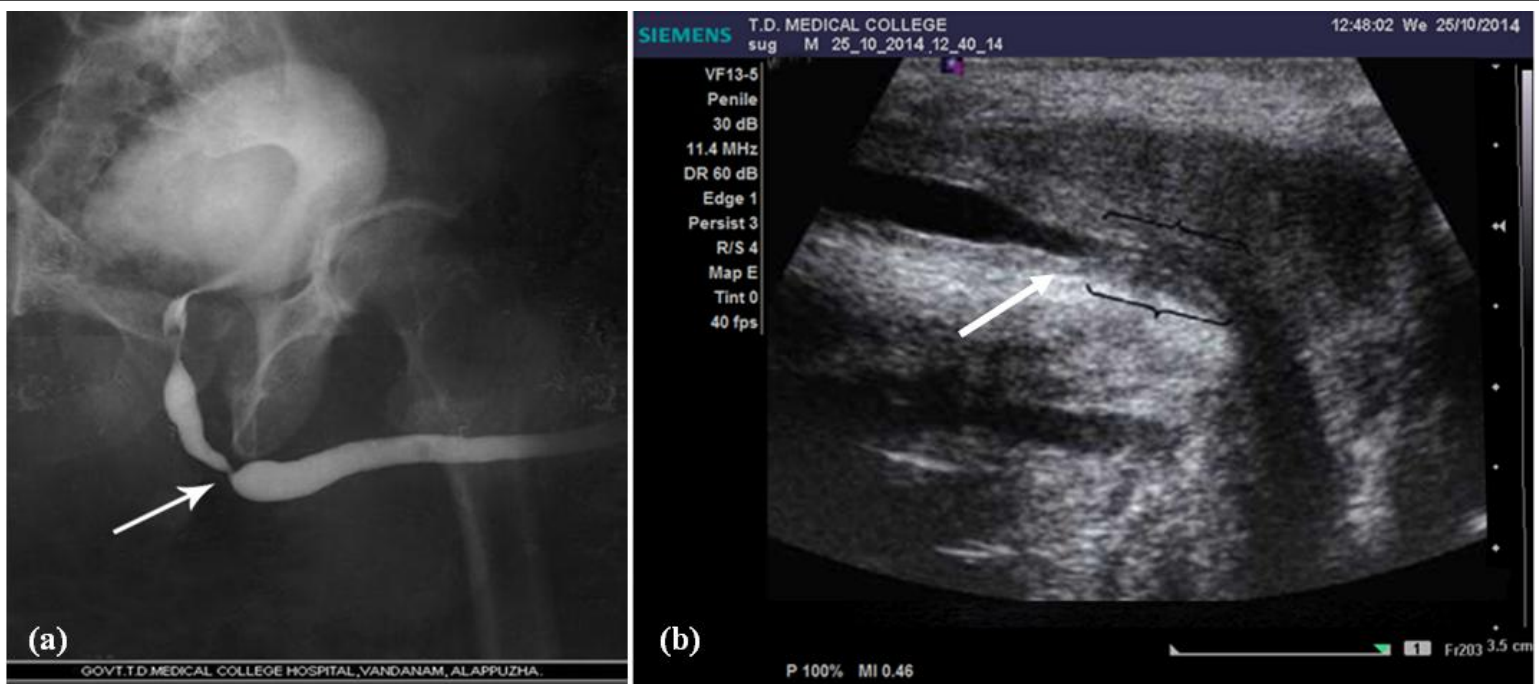

Figure 1: (a) Retrograde urethrogram showing stricture (white arrow) involving bulbar urethra.(b) Same patient underwent sonourethrography, which showed exact stricture length (brackets)involving bulbar urethra with associated spongiofibrosis (white arrow)

\section{DISCUSSION}

Blunt injuries, urinary tract infections and sexually transmitted diseases were the leading causes of anterior urethral pathologies. However, with the advent of early and effective antibiotic therapy, the incidence of urethral strictures due to infections is on a downhill. In the modern era blunt trauma is by far the leading cause of anterior urethral strictures ${ }^{2,3}$. This fact was substantiated in this study which showed trauma to be the leading cause of anterior urethral strictures accounted for $83 \%$ in this study.

Dynamic retrograde urethrography had been the 'gold standard' in the investigation of male urethra $^{15}$. This is supplemented by a voiding urethrography in case of a total stricture to delineate the proximal extent. Rarely, is the posterior urethra seen adequately if the contrast medium is not flowing through the posterior urethra at the time of exposure, because of the 'milk back action of the intrinsic sphincter which maintains the posterior urethra empty of contents. Sonourethrography is an alternative technique for evaluation of pathologies isolated to the anterior urethra. The major objective of this study was to characterize the stricture by both conventional radiography and sonourethrography. Sonourethrography detected all the strictures, which were detected on conventional radiography. In a study conducted by B.R. Ravikumar et al, sensitivity, specificity, positive predictive value, negative predictive value in anterior urethral stricture detection by sonourethrogram were found to be $100 \%{ }^{2}$. $100 \%$ sensitivity, specificity, positive predictive value, and negative predictive value of sonourethrogram was substantiated in this study also in the case of detection of anterior urethral strictures. On conventional radiography, the strictures were characterized based on two factors, that is, site and length of the stricture. Sonourethrographic characterization of strictures was based on four factors - site, length, degree of occlusion and periurethral spongiofibrosis. Conventional radiography and sonourethrography showed no disparity in the site of the stricture. However, there were significant differences in the length of the stricture assessed by both the modalities. The urethral ultrasound was more accurate because the handheld transducer was positioned in the mid-saggital plane, directly perpendicular to the diseased urethral segment. During standard retrograde urethrography the pelvis was aligned obliquely with respect to the anteroposterior xray beam and the bulbar portion of the urethra was fixed in the same axis as the pelvis. As a result the radiographic view was an 'end on' view which reduced apparent stricture length. In two patients, the stricture involved the 
bulbomembranous segment extend proximally and the posterior extent of the stricture could not be delineated by sonourethrography. As per literature, sonourethrography provides a more accurate assessment of stricture length when it is confined to the anterior urethra ${ }^{16}$. In this study Intraclass Correlation Coefficient of 0.981 between Retrograde urethrogram and Sono urethrogram in the assessment of stricture length was obtained. However, Cohen's kappa level of agreement between Retrograde urethrogram and Sono urethrogram in the assessment of stricture length was only 0.447 which was only moderate in agreement, probably because Retrograde urethrography has under estimated the stricture length in many cases. So it is likely that sonourethrography was more correct in assessing the stricture length than retrograde urethrography. But the same could not be established with this study because of the lack of a perfect reference, which is unlikely to give the 'absolute truth' about the length of the stricture. However when the stricture extends into the posterior urethra, the stricture is assessed in its entirety by radiographic urethrography while ultrasound does not delineate its posterior extent. The degree of stricture was assessed by ultrasound on cross sectional images. Conventional radiography did not provide crosssectional images, so only an arbitrary assessment of the degree of occlusion could be made by visual perception. So the degree of stricture was not assessed on conventional radiography. Radiographic urethrography provided no information with regard to the periurethral fibrotic changes. This was a definite advantage of sonourethrography. On ultrasound, severe spongiofibrosis was easily detected by the high reflectivity and posterior shadowing and the objective criteria of distensibility of less than $3 \mathrm{~mm}$. However, differentiation between normal to mild and mild to moderate spongiofibrosis was subjective based on visual perception and was not always accurate. Sonourethrography provided a more complete characterization of the stricture and defining surgical options based on these criteria's result in a low rate of recurrence. Advantages of sonourethrography included the pathology can be assessed in all the three dimensions, Accurate assessment of stricture length and assessment of periurethral fibrosis. Also there was no radiation exposure, thus sonourethrography does not cause any harmful effects to the patient and the radiologist. Since the procedure is performed with normal saline, there is no risk of contrast reaction.

\section{CONCLUSION}

The anterior urethra due to its superficial location is easily amenable to ultrasonography. The study revealed that the sonourethrography was positive in all cases in which retrograde urethrography also showed strictures. Thus, ultrasound was $100 \%$ sensitive and specific in the detection of anterior urethral strictures, taking RGU as the reference standard. Blunt trauma was the leading cause of anterior urethral strictures. The bulbar urethral strictures were often shorter on radiography when compared to sonourethrography. A good Intraclass Correlation Coefficient (0.981) between retrograde urethrography and sono urethrography in the assessment of stricture length was obtained in the study. Measure of agreement of kappa in the assessment of stricture length between sonourethrography and retrograde urethrography was moderate (0.447). Sonourethrographic characterization of the stricture, by accurately assessing the site, length, degree of occlusion and periurethral spongiofibrosis provides a more complete information for the surgeon to plan the right surgical option. The choice of the right surgical option greatly reduces the chance of recurrence. However when there is extension of the stricture to the posterior urethra or in isolated posterior urethral strictures, dynamic retrograde urethrography is still the gold standard.

Funding: Nil, Conflict of interest: None .Permission of IRB: Yes 


\section{REFERENCES}

1. Jordan GH, Schlossberg SM, Devine C.J: surgery of the penis and urethra. Campbell's Urology, 7th edn, W. B. Saunders company, 1998; 3341-69.

2. Ravikumar BR, Tejus C, Madappa KM, Prashant D, Dhayanand GS. A comparative study of ascending urethrogram and sonourethrogram in the evaluation of stricture urethra, IBJU, March - April, 2015; 41 (2): 388-392.

3. Morey AF, McAninch JW. Sonographic staging of anterior urethral strictures. J Urol 2000;163:1070-5.

4. Sandler MC, McCallum WR, Injuries of the urethra Clinical Urogrphy. Howard. M.Pollock,Vol 2,W.B.Saunders Company : 1990;1522-34.

5. Gluck CD, Bundy Al et al. Sonographic Urethrogram: Comparison to roentgenographic techniques in 22 patients. J. Urol, 1988 Dec; 140(6): 1404-8.

6. McAninch JW, Laing FC, Jeffrey B. Sonourethrography in the evaluation of urethral strictures. J. Urol. 1988 Feb; 139(2): 294-7.

7. Merkle W, Wagner W. Sonography of distal male urethra- a new diagnostic procedure for urethral strictures, J. Urol, 1988; 140: 1409-11.

8. Devine CP, Devin CJ, Jr: Operations for urethral stricture-Stewart's Operative Urology, 2nd edn, Williams and Wilkins, 1989; 650-80.

9. Kulkarni S, Barbagli G: Art of Urethral Reconstruction Ist Edn. Elsevier,2012; 234-35.

10. Blandy J: Operations of the male urethra. Operative urology, 2nd edn., Blackwell Scientific Publications, 1986 ; 206-37

11. Pierce JM Jr: Management of dismemberment of the prostatomembranous urethra and ensuring stricture disease. J. Urol. 1972; 107: 259-64.

12. Pushkarna R, Bhargava SK, Jain M: Ultrasonographic evaluation of male anterior urethra, Indian Journal of Radiology \&Imaging 2000 May; 10(2): 89-91.

13. Morey AF, McAninch JW. Sonographic staging of anterior urethral strictures. J Urol 2000;163:1070-5.

14. McAninch JW, Laing CF, Klosterman WP: Sonourethrography in the evaluation of urethral stricture disease. Urologic Clinics of North America 1989; 16(4): 791-97.

15. Rickards D, Jones S, The UrethraTextbook of Radiology and Imaging. David Sutton, VI edno, Vol.2, Churchill Livingstone, 1998; 1189-95.

16. Morey AF, McAninch JW. Role of preoperative sonourethrography in bulbar urethral reconstruction. J Urol 1997;158:1376-9. 